\title{
The Application of Computed Tomography in the Prevention of Ovarian Cancer
}

\author{
Lihong Bao
}

Medical College, Inner Mongolia University for the Nationalities, Tongliao, Inner Mongolia, 028000, China

baolihong71@163.com

\begin{abstract}
CT and ultrasound as the examination means are commonly used in clinical diagnosis of ovarian cancer, because of the characteristics of CT and its wide range of scanning, faster image acquisition speed, it is one important examination means for ovarian cancer diagnosis and staging. In this paper, it takes the efficacy of CT in ovarian cancer prevention as the starting point, with the help of the manifestation of CT of ovarian cancer that is commonly described, discussing the pathological basis of ovarian cancer and the recurrent diagnosis CT of ovarian cancer.
\end{abstract}

\section{Introduction}

Epithelial ovarian cancer is a major health problem and represents 40 to 50\%of coloepithelial ovarian cancer. Epithelial ovarian cancer is one of the leading causes of cancer deaths in the world. The improvement of computerized Tomography (CT), gave us new possibilities for diagnosing coloEpithelial ovarian cancer. For successful execution of surgical techniques, good diagnostic imaging of the cancer is necessary in order to have a low level of recurrence. In this study, we have done study blood supply of the preserved image based model obtained from CT. Epithelial ovarian cancer ranks the first in malignant tumors with high rate of gynecological cancer incidence and mortality, especially the rate of occurring high class differentiation of epithelial ovarian cancer incidence is very high, accounting for $65 \%$ of the total number of ovarian cancer. Because it is located in the deep ovarian pelvic, in the early stage of tumor, patients are often lack of features. When the obvious signs occurred and went to have treatment, who often have suffered the advanced cancer in the late stage. Therefore, the early diagnosis of ovarian cancer has important clinical significance for improving the survival rate of patients.

\section{The Efficacy in the Prevention and Treatment of CT in Ovarian Cancer}

CT can clearly display the anatomical structure of the pelvic organs, which also can determine the location of the tumor and cystic features, finding out the invasion of adjacent tissue and other abdominal organs, as well as lymph node metastasis, moreover, it can carry out needle biopsy under CT guidance, so as to improve the diagnosis rate of ovarian tumor. Normal ovarian CT often show low density on both sides of the uterus oval, smooth edges, uniform density, non enhancing. The diagnostic criteria of CT ovarian carcinoma: tumor is mainly with cystic, solid or completely solid, after the contrast enhancement, the solid part can be enhanced; cyst spacer thickness is more than $3 \mathrm{~mm}$, the visible capsule wall stick out of the capsule is nodular mass; the visible mass of irregular necrosis can be seen, besides, there is no contrast enhancement; abdominal cavity and viscera can see the metastatic nodules; with ascites fluid, abdominal and pelvic lymph nodes are more than $1 \mathrm{~cm}$.

The staging diagnosis standard of CT can carry out the diagnosis according to FIGO staging diagnosis of ovarian cancer after the revised CT and MRI in 1986. S. Guerriero and other staff made research and showed that, CT diagnosis can better make position for ovarian tumors, besides, the accurate degree is very high in the diagnosis of cystic teratoma, but for the identification of benign and malignant ovarian tumors, the accuracy is lower than that of $\mathrm{B}$ ultrasound. The domestic research thought: the detection of malignant peritoneal, mesenteric or omental implantation, lymph node enlargement (metastasis) and other features of CT is significantly higher than that of B 
ultrasound.moreover, in the qualitative diagnosis of ovarian tumors, as well as the aspect of the assisted clinical stage of malignant tumor, it is better than B ultrasound.

CT nano contrast agent is a hot spot research in recent years, because the particle size is nanometer, so the biological behavior is different from the traditional iodine contrast agent. When the size of nano particle is $10-200 \mathrm{~nm}$, it can achieve the purpose of tumor targeting through the enhanced permeation and retention effect.For example, Keun and the other scholars introduced CT imaging to SCC-7 target cells and multi-functional nano particles with fluorescence molecular by adopting temperature induced phase method, but due to the phagocytic effect of liver, spleen and other tissues, as well as the non- stability, immunogenicity and other reasons, in fact, the targeting ability of this kind of targeting nano particles is not as high as expected. Besides, the above reports are nano particles by changing the particle size to achieve passive targeting purposes, the control on the size is very stringent, at the same time, the requirements on the synthesis technology are relatively high, besides, by adopting this kind of method, the accuracy of preparing nano particles for the tumor targeting sites is not high.

\section{The Common Manifestations of CT of Ovarian Cancer}

(1)Firstly, pelvic mass, which is mainly cystic solid, often involving the bilateral sides, besides, there are more solid soft tissue components, which showed small nodular or papillae, ranging in size, if there is more solid components, the degree of malignancy will be higher; (2) Secondly, the internal solid mass exists low density necrosis zone, or see enlarged vessels; (3) Thirdly, the tumor is cystic structure, but the thickness of cystic wall and septum is uneven, the thickest place is more than 3mm; (4) Fourthly, the enhanced tumor wall nodules and mass parenchyma have obvious enhancement effect, CT value is more than 30HU; (5) Lastly, the signs of metastasis: the metastasis of wall layer of peritoneal metastasis are usually local thickening, with wide band, nodular or mass like. Omental metastases mostly have the transverse and anterior abdominal wall nodular, the shape of it is like a pie. As for liver metastasis, mostly low density nodules are existed with part of calcification. Retroperitoneal lymph node metastasis, the lymph node metastasis is increased with central necrosis. If the mentioned phenomena are occurred it can have a definitive diagnosis.

Table 1 Types of Tumor and LDH

\begin{tabular}{cccc}
\hline $\begin{array}{c}\text { Types of Tumor } \\
\text { Blood }\end{array}$ & $\mathrm{n}$ & LDH $\overline{\mathrm{x}} \pm \mathrm{s}(\mathrm{U} / \mathrm{L})$ & Blood CA125 $\overline{\mathrm{x}} \pm \mathrm{s}(\mathrm{U} / \mathrm{L})$ \\
\hline $\begin{array}{c}\text { Ovarian Cancer } \\
\text { Benign Ovarian } \\
\text { Tumor }\end{array}$ & 50 & $405.5 \pm 331.5$ & $355.3 \pm 279.0$ \\
$\begin{array}{c}\text { Cervical Cancer } \\
\text { and Endometrial } \\
\text { Cancer }\end{array}$ & 22 & $205.0 \pm 70.5$ & $27.2 \pm 20.9$ \\
\hline
\end{tabular}

\section{The Pathological Basis}

Ovarian cancer is derived from epithelial malignant tumor, the common pathological type is as follows: liquid cystadenocarcinoma, mucinous cystadenocarcinoma. As for liquid cystadenocarcinoma, the diameter of tumor can reach $50 \mathrm{~cm}$, with smooth surface or papillary growth. Section has more room with the real cystic, necrosis, hemorrhage, the cavity can be filled with papillations. As for mucinous cystadenocarcinoma, the diameter of tumor is $15-30 \mathrm{~cm}$, the cystic wall is visible with papillae or parenchyma. Section is half bag with half solid. Microscopically watched, the epithelial lining layer of cystadenocarcinoma is complex with severe atypical hyperplasia, while the cystic wall is covered with the nipples, the branches of nipples are lush, cystic part secretion with the formation of cystic tumor or part of the tumor; in tumor, there are papillary clusters, each is bypassed or nipples are disappeared, which is composed of irregular solid sheets or strips of the nests,forming the part of solid mass or masses. Therefore, CT showed cystic, 
cystic and solid tumors. In the late stage, with the metastasis of omentum, peritoneum and other organs. The signs of CT that can indicate metastasis are as follows: (1)The peritoneal surface is visible with the size of nodular tumor foci and the nodules in the backdrop of ascites is less than $1 \mathrm{~cm}$. (2) The omentum and peritoneal metastasis showed the changes in omental shape, and the local part is fixed. (3)The lymph node metastasis has the abdominal or pelvic blood vessels, retroperitoneal solid mass, no enhancement with vascular differentiation.

Table 2 The Distribution of Pathological Type

\begin{tabular}{ccc}
\hline Pathological Type & The number of cases (cases) & The proportion (\%) \\
\hline $\begin{array}{c}\text { Serous Adenocarcinoma } \\
\text { Cancer }\end{array}$ & 85 & 72.65 \\
$\begin{array}{c}\text { Mucinous adenocarcinoma } \\
\text { Cancer }\end{array}$ & 15 & 12.82 \\
$\begin{array}{c}\text { Endometrial Cancer } \\
\text { Mixed Epithelial Carcinoma } \\
\text { Cancer }\end{array}$ & 9 & 7.69 \\
\hline
\end{tabular}

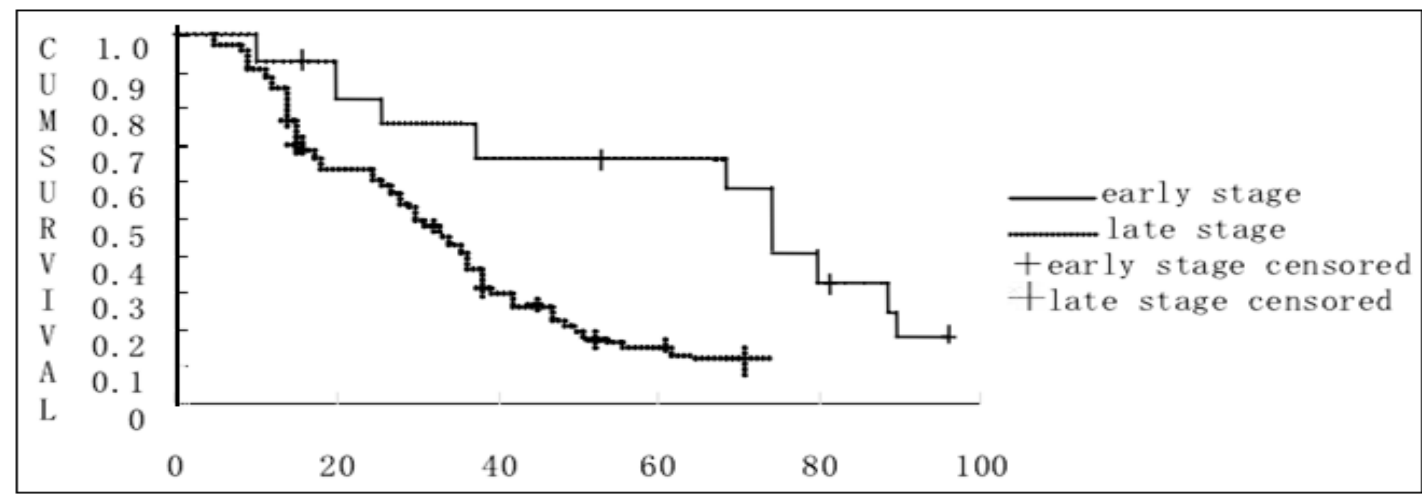

Fig.1 The Survival Curves of the Early and Late Stage

\section{CT Diagnosis of Recurrent Ovarian Cancer}

After the operation of the primary ovarian carcinoma resection and chemotherapy operation, in order to understand whether there is metastasis or not, it usually is required to use laparotomy to determine the presence of residual disease. If CT can detect the clear metastasis, then the indications of laparotomy is limited, especially those areas that can not be cleared up (such as the retroperitoneal lymph node, liver, pleural lesions).

\section{Conclusion}

Although the findings of CT examination can have radical treatment on ovarian cancer, the local part still can have recurrence. Ovarian malignant tumor abdominal dissemination can be accurately tracked by CT, but small peritoneal metastasis may be missed, a negative CT scan can not exclude the existence of intra-abdominal secondary tumors. There are some limitations in the use of CT in the early stage of ovarian cancer, while ultrasound can demonstrate the increased smaller ovary and adhesion in clusters of tumors, so as to differentiate uterine, at this point, it is better than CT, however, CT can define the whole range of disease, which also can have the diagnosis of the secondary cavity metastasis.

\section{Acknowledgments}

This paper was supported by the 2015 Inner Mongolia Higher Science Project: "Nerve growth factor and its receptor in ovarian tissue" (NJZY171) 


\section{Reference}

[1]Thomassin-Naggara I, Dara E, Cuenod CA, et al. 2008. Dynamic contrast-enhanced magnetic resonance imaging: a usefultool for characterizing ovarianepithelial tumors. J Magn Reson Imaging. vol.28, pp111-120.

[2]Sala E, Kataoka MY, Priest AN, et al. 2012. Advanced ovarian cancer: multiparametric MR imaging demonstrates response and metastasis specific effects.Radiology. vol.263, pp149-159.

[3]Takeuchi M, Matsuzaki K, Nishitani H. 2010. Diffusion-weighted magnetic resonance imaging of ovarian tumors: differentiation of benign and malignant solid components of ovarian masses. $\mathrm{J}$ Comput Assist Tomogrogy. vol.34, pp173-176.

[4]Espada M, Garcia-Flores JR, Jimenez M, et al. 2013. Diffusion-weighted magnetic resonance imaging evaluation of intra-abdominal sites of implants to predict likelihood of suboptimal cytoreductive surgery in patients with ovarian carcinoma.European Radiology. vol.23, pp2636-2642.

[5] Jemal A, Siegel R, Ward E. 2010. Cancer statistics. CA Cancer J Clin, vol.60, pp277-300. 